\title{
A comparison of inexpensive statistical packages for Apple II microcomputers
}

\author{
DARRELL L. BUTLER and STEVE K. JONES \\ Ball State University, Muncie, Indiana
}

\begin{abstract}
The purpose of this paper is to describe and compare some inexpensive software packages that calculate a variety of statistics on the Apple II microcomputer. For each package, hardware requirements, program capacity, limitations, constraints, accuracy, editing, error handling, and other features were studied.
\end{abstract}

The purpose of this paper is to compare inexpensive (less than \$100) statistical packages that run on Apple II microcomputers. Several sources were used to find packages, including previous reviews (e.g., Butler, 1986; Henry \& Bauer, 1986), advertisements in numerous journals (e.g., Amstat News), and listings of programs (e.g., Datapro/McGraw-Hill, 1985). Packages that had too little flexibility (e.g., packages from Dynacomp and PARsoft) were not included. Two of the packages found are no longer on the market: Ustats (Wm. C. Brown Publishers) and Statistics 3.0 (Eduware). Some packages could not be obtained. For unobtained packages, the information included in the present paper is brief (because it is based upon advertisements), and those packages are not included in Table 1, the primary summary of the reviewed packages.

\section{GENERAL CHARACTERISTICS OF PACKAGES}

Table 1 summarizes a variety of information about the packages. The features included in the table are described below. For each package, characteristics that are difficult to include in the table are described following the description of tabled features.

\section{General Features}

All of the packages are menu driven, at least for procedure selection. The hardware requirements of the packages are very similar. All worked on an Apple II+, Apple IIe, and Franklin 1200, although some subtle differences in operation were found. Copy-protected programs could not be copied with the standard DOS 3.3 copy program or the Pascal Filer. Error trapping refers to the program containing checks for such conditions as divide by zero and non-numeric data and appropriate handling of such conditions.

Requests for reprints should be sent to the first author at the Department of Psychological Sciences, Ball State University, Muncie, IN 47306.

\section{Statistics}

The statistics calculated by the programs were compared to a list of fundamental statistical routines, those found in at least 10 of 20 general statistics textbooks we examined. The fundamental routines are those listed in Table 1. For each statistical routine, the programs' limitations were studied: maximum number of scores, maximum number of cells or groups (where appropriate), and accuracy. Accuracy was examined using the basic technique described by Butler and Eamon (1985). This technique is particularly sensitive to rounding errors, especially those resulting from computing a power of a large number. A number of similar data sets were used. The table shows the maximum number of digits that could be used in any of the data sets to obtain correct statistics.

Speed was not easy to assess. Overall, Statistix was the fastest and Statistics Software for Microcomputers was the slowest. Speeds of the other three programs were approximately the same. However, speed depended upon length of the data set, statistic used, options, and number of consecutive runs of a procedure (i.e., most routines were slower the first time they were run).

\section{Other Features}

Disk files for all packages were sequential files. All had files with names, structures, or contents that were unique, or specific to the package. Usually these unique files were compatible with a variety of routines within the package. Some of the packages could also save ASCII data-only files or other types of files that could be used by other programs. Statistix's saved data structure contains "dummy" or "grouping" variables as well as data.

Data editing is best when data are numbered and editing is done onscreen, which means the data is seen during the editing. Transforming variables means to change data values using algebraic expressions.

\section{UNIQUE PROGRAM CHARACTERISTICS}

Those characteristics of the programs that are difficult to describe in Table 1 are described below, package by package. Addresses for suppliers of the programs are provided in the Appendix. 
Table 1

Requirements, Features, and Limitations of Programs

\begin{tabular}{|c|c|c|c|c|c|}
\hline & \multicolumn{5}{|c|}{ Programs } \\
\hline & Keystat & $\begin{array}{l}\text { Statistical } \\
\text { Programs }\end{array}$ & $\begin{array}{l}\text { Statistical } \\
\text { Software }\end{array}$ & $\begin{array}{l}\text { Statistics with } \\
\text { Interpretations }\end{array}$ & Statistix \\
\hline \multicolumn{6}{|c|}{ GENERAL FEATURES } \\
\hline \multicolumn{6}{|l|}{ Hardware } \\
\hline RAM & $48 \mathrm{~K}$ & $48 \mathrm{~K}$ & $48 \mathrm{~K}$ & $48 \mathrm{~K}$ & $64 K$ \\
\hline Minimum Number Disk Drives & 1 & 1 & 1 & 1 & 2 \\
\hline 80-Column Card & No & No & Yes & No & Optional \\
\hline Printer & Optional & Optional & Optional & Optional & Optional \\
\hline Cost & $\$ 50 / \$ 10$ & Free & $\$ 26$ & $\$ 25$ & $\$ 75$ \\
\hline Menus & Yes & Yes & Yes & Yes & Yes \\
\hline Copy Protected & Yes & No & No & No & No \\
\hline List Protected & Yes & No & No & No & Yes \\
\hline Language & Unknown & BASIC & Unknown & BASIC & Pascal \\
\hline Manual & No & No & Yes & No & Yes \\
\hline Overall Error Trapping Good & No & No & No & Yes & Yes \\
\hline \multicolumn{6}{|c|}{ STATISTICS } \\
\hline \multicolumn{6}{|l|}{ Descriptive Statistics } \\
\hline Number of Cases & 200 & 2,100 & 100 & 2,000 & 3,000 \\
\hline Mean (Accuracy) & Yes (9) & Yes (8) & Yes (7) & Yes (9) & Yes (6) \\
\hline Median (Accuracy) & Yes (9) & No & Yes (7) & Yes (9) & No \\
\hline Mode (Accuracy) & No & No & Yes $(0)$ & Yes (9) & No \\
\hline Variance (Accuracy) & Yes (9) & Yes (3) & Yes (3) & Yes (8) & Yes (6) \\
\hline Standard Deviation (Accuracy) & Yes (9) & Yes (3) & Yes (3) & Yes (8) & Yes (6) \\
\hline Min, Max (Accuracy) & No & No & Yes (7) & Yes (9) & Yes (6) \\
\hline \multicolumn{6}{|l|}{ Correlation/Regression } \\
\hline Pearson $r$ & Yes & Yes & Yes & Yes & Yes \\
\hline Number of Cases & 500 & 2,050 & 239 & 2,000 & 3,000 \\
\hline Accuracy & 9 & 3 & 4 & 6 & 5 \\
\hline Spearman tho & No & Yes & No & Yes & Yes \\
\hline Number of Cases & - & 2,050 & $\rightarrow$ & 1,000 & 3,000 \\
\hline Accuracy & - & 3 & - & 9 & 6 \\
\hline Simple Linear Regression & Yes & Yes & Yes & Yes & Yes \\
\hline Number of Cases & 500 & 2,000 & 239 & 2,000 & 3,000 \\
\hline \multicolumn{6}{|l|}{ Significance Tests } \\
\hline$t$ Test (One Group) & Yes & No & Yes & No & Yes \\
\hline Number of Cases & 200 & - & $10^{7}$ & - & 3,000 \\
\hline Accuracy & 9 & - & 3 & - & 4 \\
\hline$t$ Test (Independent Groups) & Yes & Yes & Yes & Yes & Yes \\
\hline Number of Cases & 300 & 2,400 & $10^{7}$ & 3,000 & 3,000 \\
\hline Accuracy & 5 & 3 & 3 & 8 & 4 \\
\hline$t$ Test (Dependent Groups) & Yes & Yes & Yes & Yes & Yes \\
\hline Number of Cases & 100 & 1,200 & $10^{7}$ & 3,000 & 3,000 \\
\hline Accuracy & 9 & 3 & 2 & 8 & 4 \\
\hline Mann-Whitney $U$ & Yes & Yes & No & Yes & Yes \\
\hline Number of Cases & 200 & 1,400 & - & 2,000 & 3,000 \\
\hline Accuracy & 9 & 4 & - & 9 & 9 \\
\hline Wilcoxon & Yes & Yes & No & Yes & Yes \\
\hline Number of Cases & 100 & 800 & - & 2,000 & 3,000 \\
\hline Accuracy & 9 & 4 & - & 9 & 9 \\
\hline ANOVA (One-Way Between) & Yes & Yes & Yes & Yes & Yes \\
\hline Number of Levels & 9 & Dep & Dep & 100 & 30 \\
\hline Total Number of Cases & 400 & Dep & Dep & 3,000 & 90,000 \\
\hline Maximum Cases Per Level & 50 & Dep & Dep & 2,998 & 3,000 \\
\hline Accuracy & 6 & 4 & 6 & 8 & 7 \\
\hline ANOVA (One-Way Within) & Yes & Yes & Yes & Yes & Yes \\
\hline Number of Levels & 9 & Dep & Dep & 100 & 20 \\
\hline Maximum Number of Cases & 9 & Dep & Dep & 2,000 & 3,000 \\
\hline Accuracy & 3 & 4 & 5 & 8 & 3 \\
\hline ANOVA (Two-Way Between) & Yes & Yes & Yes & No & Yes \\
\hline Number of Levels & $9 * 9$ & $9 * 9$ & Dep & - & $20 * 20$ \\
\hline Total Number of Cases & 729 & 2,025 & Dep & - & 3,000 \\
\hline Maximum Cases per Level & 9 & 25 & Dep & - & 2,998 \\
\hline Accuracy & 3 & 4 & 5 & - & 3 \\
\hline
\end{tabular}


Table 1 (Continued)

\begin{tabular}{|c|c|c|c|c|c|}
\hline Kruskal-Wallis & Yes & No & No & Yes & Yes \\
\hline Number of Levels & 9 & - & - & 100 & 30 \\
\hline Total Number of Cases & 450 & - & - & 2,000 & $? ?$ \\
\hline Maximum Cases per Level & 50 & - & - & 1,998 & 3,000 \\
\hline Accuracy & 9 & - & - & 9 & 9 \\
\hline Friedman & Yes & No & No & Yes & Yes \\
\hline Number of Levels & $9 * 9$ & - & - & 500 & $30 * 30$ \\
\hline Total Number of Cases & 729 & - & - & 2,000 & 3,000 \\
\hline Maximum Cases per Level & 9 & - & - & 1,998 & 2,998 \\
\hline Accuracy & 9 & - & - & 9 & 9 \\
\hline Chi-Square Goodness of Fit & Yes & No & No & Yes & No \\
\hline Number of Levels & 100 & - & - & 2,000 & - \\
\hline Maximum Frequency & $10^{9}$ & - & - & $10^{\circ}$ & - \\
\hline Accuracy & 9 & - & - & 8 & - \\
\hline Chi-Square Contingency & Yes & No & Yes & Yes & Yes \\
\hline Number of Levels & $9 * 9$ & - & -100 & $45 * 45$ & $30 * 30$ \\
\hline Maximum Frequency & $10^{\circ}$ & - & $? ?$ & $10^{9}$ & $?$ \\
\hline Accuracy & 8 & - & 1 & 8 & 9 \\
\hline \multicolumn{6}{|c|}{ OTHER FEATURES } \\
\hline \multicolumn{6}{|l|}{ Graphics } \\
\hline Scattergram & Yes & No & No & No & Yes \\
\hline Histogram & No & No & No & No & Yes \\
\hline Data Input Disk Files & Yes & Yes & Yes & Yes & Yes \\
\hline Specific to Package & Yes & No & Yes & Yes & Yes \\
\hline Compatible Across Routines & No & Yes & Yes & Yes & Yes \\
\hline Other (e.g., ASCII) & No & Yes & No & No & Yes \\
\hline Grouping Variables Needed & No & No & No & No & Yes \\
\hline Good Error Trapping & No & No & No & Yes & Yes \\
\hline Data Editing & Yes & Yes & Yes & Yes & Yes \\
\hline Onscreen & No & No & No & Yes & No \\
\hline Cases Numbered & Yes & No & Yes & Yes & Yes \\
\hline Good Error Trapping & No & No & No & Yes & Yes \\
\hline Transforming Variables & Log & No & No & Log & Extensive \\
\hline
\end{tabular}

\section{Key-Stat}

The correlation/regression program is a strength of this package. Output of the regression routine includes descriptive statistics and many intermediate values used in calculating the correlation coefficient. A scatter plot and regression equation are generated. The best-fitting line can be added to the scatter plot. (Note, the scatter plot is accurate only when the data have four digits or less.)

In addition to the statistics listed in Table 1, Key-Stat has a rather elaborate calculator that simulates a HewlettPackard RPN calculator.

Some of the menus (especially the opening menu) are long and use confusing terminology. As a result, it is difficult to find some statistical routines. To aid the user, a separate program is included that helps the user to choose the correct statistical technique. Another inconvenience not apparent in Table 1 is that the user must return to the main disk menu before rerunning some of the procedures.

For another review of Key-Stat, see Henry and Bauer (1986).

\section{Statistical Programs for the Apple II}

In the ANOVA routines, the number of levels of independent variables and the number of scores per level are interdependent. For example, a one-way between design with two levels accepts $\mathbf{5 0 0}$ scores per level, a one-way with four levels accepts 150 scores per level, and a oneway with 20 levels accepts only 7 scores per level.

In addition to the statistics listed in Table 1, this package calculates three-way ANOVAS, simple Latin square ANOVAS, and ANCOVAS with a maximum of 100 cells and 25 scores per cell. The ANOVA routine is accurate to three digits.

The program was originally described in Steinmetz, Romano, and Patterson (1981). Several procedures have been added since the original publication.

\section{Statistics Software for Microcomputers}

In the ANOVA routines, the total number of scores allowed is dependent upon the number of cells in the design. For example, a one-way between design with two levels accepts 3,450 total scores, a one-way with five levels accepts 1,700 total scores, and a one-way with 15 levels accepts 625 total scores.

In addition to the statistics listed in Table 1, this package can compute multilinear regression and factor analysis.

The program's greatest weakness is in the data input routines. The input routines vary with procedure and all 
are very slow. The user is forced to start a routine over if any error is made. With the exception of the excellent editor for the descriptive routines, editing is difficult and the chi-square routine does not allow editing. Calculations are very sluggish. For example, the descriptive statistics program requires approximately $10 \mathrm{~min}$ to calculate statistics on 200 data. Note that Table 1 indicates that the descriptive statistics can only handle 100 values. That is the number of values the program can process in $3 \mathrm{~min}$. A more patient user may find that the program has great capacity.

\section{Statistics with Interpretations}

One strength of this package is the output. It includes the statistics and, where appropriate, a verbal statement indicating whether the statistic is significant, the degrees of freedom, the probability of the statistic, and a list of the assumptions of the test.

In addition to the statistics listed in Table 1, Statistics with Interpretations calculates skewness, kurtosis, Cramér's V for contingency tables, and multiple regression. However, Cramér's V is only accurate to two digits, and the multiple regression routine is limited to two predictors.

At the beginning of each statistical procedure is a list of the procedure's capacity and limitations; then the user has the option to escape, input data from the keyboard, or read data from a disk file.

\section{Statistix}

This package is the most comprehensive reviewed here. The output of several of the routines (e.g., ANOVA) is far more complete than any other package reviewed here, and many more options are available (e.g., contrasts). In addition to the statistics described in Table 1, this package also computes the following statistical procedures: ANOVA (up to five independent variables), ANCOVA (up to five covariants), principal components (up to 30 variables), multiple regression (up to 30 predictors), sign test, median test, Kolmogorov Smirnov test, log linear models, McNemar's symmetry test, 11 different statistics on $2 \times 2$ tables, the runs test, Wilk-Shapiro/Rankit Plots, and several types of cross tabulations. There is also an extensive procedure for calculating probabilities.

This package is relatively easy to use, but is a bit more difficult to use than the other packages reviewed here. For example, the ANOVA routines require the user to specify the model and indicate the error term(s). A splitplot factorial design may be specified as follows:

$$
D V=A B A^{*} B(E R R O R) C C * B(E R R O R) A^{*} C A^{*} B^{*} C(E R R O R),
$$

where $A, B$, and $C$ are dummy variables specifying group membership and DV is the data to be analyzed.

For another review of the package, see Russek-Cohen (1986).

Psychostat-3 (and newer version called Apstat)

Several telephone calls and a written communication to this company were made requesting a review copy. In addition, I offered to purchase a copy if they would refund my money after the review. The company did not cooperate. As a result, the following comments are based upon advertisements of this package.

This package runs on a one-drive Apple II or compatible. It costs \$99. Calculations include descriptive statistics, $t$ tests, ANOVAs (up to five factors and unlimited cases), multiple regressions (up to 25 predictors), and nonparametric statistics. Data editing, transformations, file compatibility with other packages, and graphics (bargraphs and scattergrams) are included in this menu-driven program.

\section{Statcalc}

This package costs $\$ 100$. We did not test it, because no copy was received. However, it is a command language program that accommodates 2,000 data on a $48 \mathrm{~K}$, one-drive Apple. There are seven types of commands: (1) input, editing, and display of data; (2) means, variances, $t$ tests, chi-squares, ranks; (3) plots: scatter plot, triplots, stem and leaf, boxplots, normal plots, histograms; (4) transform and sorting; (5) random number generation; (6) one- and two-way ANOVA, regression, multiple regression; and (7) DOS commands.

\section{REFERENCES}

BUTLER, D. L. (1986). Elementary statistics packages for microcomputers. Contemporary Psychology, 31, 485-487.

BUTLER, D. L., EAMON, D. B. (1985). An evaluation of statistical software for research and instruction. Behavior Research Methods, Instruments, \& Computers, 17, 352-358.

DAtaPro/McGraw-Hill. (1985). Guide to Apple software (2nd ed.). New York: McGraw-Hill.

HenRY, N. W., \& BAUER, D. F. (1986). Key-Stat. The American Statistician, 40, 50-51.

RussEX-CoHEN, E. (1986). Statistical package for microcomputers: Statistix 1.0. Bulletin of the Ecological Society of America, 67, 194-196.

Steinmetz, J. E., Romano, A. G., \& Patterson, M. M. (1981). Statistical programs for the Apple II microcomputer. Behavior Research Methods \& Instrumentation, 13, 702.

\section{APPENDIX \\ Program Suppliers' Addresses}

Key-Stat

Oakleaf Systems

P.O. Box 472

Decorah, IA 52107

Statistical Programs for the Apple II

Michael M. Patterson

College of Osteopathic Medicine

Ohio University

Athens, $\mathrm{OH} 45701$

(614) 593-2337

Statistics Software for Microcomputers

Kern International, Inc.

433 Washington St.

P.O. Box 1029CA

Duxbury, MA 02331

(617) 934-0445 
Statistics with Interpretations

Darrell L. Butler

Department of Psychological Science

Ball State University

Muncie, IN 47303

(317) 285-1690

Statistix

NH Analytical Software

801 W. Iowa Ave.

St. Paul, MN 55117

(612) 488-4436

Psychostat-3 (and Apstat)

Statsoft

2832 E. 10th St., Suite 4

Tulsa, OK 74104

(918) 583-4149

Statcalc

Alan J. Lee and Peter McIneruey

Department of Mathematics and Statistics

Peter R. Mullins

Department of Community Health

University of Auckland

Private Bag

Auckland, New Zealand 